\title{
Circuit
}

Musiques contemporaines

\section{Programme détaillé de Montréal/Nouvelles Musiques 2003}

Volume 14, numéro 2, 2004

Montréal/Nouvelles Musiques

URI : https://id.erudit.org/iderudit/902316ar

DOI : https://doi.org/10.7202/902316ar

Aller au sommaire du numéro

Éditeur(s)

Les Presses de l'Université de Montréal

ISSN

1183-1693 (imprimé)

1488-9692 (numérique)

Découvrir la revue

Citer ce document

(2004). Programme détaillé de Montréal/Nouvelles Musiques 2003. Circuit,

14(2), 77-82. https://doi.org/10.7202/902316ar d'utilisation que vous pouvez consulter en ligne.

https://apropos.erudit.org/fr/usagers/politique-dutilisation/ 


\section{Programme détaillé de Montréal/Nouvelles Musiques 2003}

\section{Dimanche 2 mars 2003}

"Démons et merveilles», Ensemble de la SMCQ, Walter Boudreau, dir.

Salle Pollack

George Antheil, Ballet mécanique

Michel Longtin, Pohjatuuli : hommage à Sibelius

"Petites musiques sentimentales», Yves Daoust

Salle Beverly Webster Rolph du Musée d'art contemporain de Montréal

Petite musique sentimentale, Jacques Drouin, piano

Adagio, Lise Daoust, flûte

Solo, Louis-Philippe Marsolais, cor

L'entrevue, Joseph Petric, accordéon

Impromptu, Jacques Drouin, piano, Lorraine Vaillancourt, clavier électronique

\section{Lundi 3 mars 2003}

"Architectures en mouvement», VivaVoce

Salle Redpath

Giovanni Gabrieli, O Jesu mi dulcissimi; Hodie completi sunt

André Villeneuve, Ecce ubi sum

Guillaume Dufay, Nuper rosarum flores

Brian Cherney, Tenebrae *

Morton Feldman, Rothko Chapel

"Acousmates un jour... ", Réseaux des arts médiatiques

Salle Beverly Webster Rolph du Musée d'art contemporain de Montréal

Monique Jean, Danse de l'enfant esseulée

Ned Bouhalassa, Constamment (autoportrait)

Alain Gauthier, Course-Métrage

Marc Tremblay, Musique pour un appartement meublé

Francis Dhomont, Les moirures du temps 


\section{Mardi 4 mars 2003}

"Les voix à I'honneur", Hilliard Ensemble

Salle Pollack

Piers Hellawell, The Hilliard Songbook

Rudolf Kelterborn, Four Sonnets for Four Voices

Elizabeth Liddle, Whale Rant

José Evangelista, Songs of Innocence and of Experience *

Paul Steenhuisen, Les enfers éternels des gens désespérés *

Joanne Metcalf, II nome del bel fior

Arvo Pärt, And One of the Pharisees

Alexander Raskatov, Praise

\section{Mercredi 5 mars 2003}

"Bloed / Noces de sang 》, Hilliard Ensemble et McGill Contemporary Music Ensemble, Denys Bouliane, dir.

Salle Pollack

Cornelis de Bondt, Bloed

"No Man's Clan», Robert Cram, Lori Freedman et leurs invités

Harry Freedman, Romp and Reverie *

Niels Rosing-Schow, Epiages

Michael Jarrell, Assonance II

Bruce Mather, Doisy Daëne

Omar Daniel, Dreams of the Panther

Michel Gonneville, Attiré vers le haut par le menu

Lori Freedman, No Man's Clan

\section{Jeudi 6 mars 2003}

"Montréal a rendez-vous avec Vienne», Kangforum Wien

Salle Pierre-Mercure

Georg Friedrich Haas, Tria ex uno; Hommage à György Ligeti

Yannick Plamondon, Autoportrait sur Times Square

André Ristic, Sonate de carnaval *

Bernhard Lang, Schrift-Bild-Schrift

"Nouvelle musique d'hiver», Ensemble SuperMusique, Joane Hétu

Salle Beverly Webster Rolph du Musée d'art contemporain de Montréal 


\section{Vendredi 7 mars 2003}

"Les temps modernes" (repris le 8 mars), Orchestre symphonique de McGill, Denys Bouliane et Alexis Hauser, dir.

Edgar Varèse, Déserts

György Ligeti, Ramifications

Dmitri Chostakovitch, Symphonie $n^{\circ} 1$ en fa mineur, op. 10

"Licks \& Brains», Ensemble de la SMCQ et le quatuor de saxophones Quasar Klas Torstensson, Tryptich Licks and Brains

\section{Samedi 8 mars 2003}

$15^{\mathrm{e}}$ Concours national des jeunes compositeurs de CBC/Radio-Canada Salle Beverly Webster Rolph du Musée d'art contemporain de Montréal Catégorie musique électroacoustique, les finalistes:

Félix Boisvert, Chpoc!

Jean-Michel Robert, Les enfants d'Éole

Louis Trottier, Nom d'une casserole

En complément de programme : Jacques Tremblay, Oaristys

"Des pirates à l'assaut de la musique contemporaine» la Nef

Salle Pierre-Mercure

Silvy Grenier, L'eau ivre

Robert Marcel Lepage, La machine à explorer le tempo

"Faus Technology», Purform (Alain Thibault, musique et Yan Breuleux, visuel) Salle Beverly Webster Rolph du Musée d'art contemporain de Montréal Faus Techonology, musique numérique, 3 lecteurs DVD et 3 écrans vidéo

\section{Dimanche 9 mars 2003}

$15^{\mathrm{e}}$ Concours national des jeunes compositeurs de CBC/Radio-Canada Salle Pierre-Mercure

Catégorie œuvres pour piano solo, les finalistes:

Charles-Antoine Fréchette, Trajectoire

Matthew Rizzuto, Perseveration Kids

Maxime Després, Les cendres d'un paysage d'encre éphémère

Catégorie musique de chambre, les finalistes:

Andriy Talpash, HWY 2

Analia Llugdar, Inflexions

Niklas Kambeitz, The Days of Victory

En complément de programme :

Ana Sokolovic, Géométrie sentimentale 


\section{Lundi 10 mars 2003}

«Triptyque intimiste», Salle Pierre-Mercure :

"Sons et lumières», Quatuor Molinari et Marie-Danielle Parent

Béla Bartók, Quatuor $n^{\circ} 3$

Sofia Gubaidulina, Quatuor no 4

R. Murray Schafer, Quatuor $n^{\circ} 7$ soprano obligato

«Et les pierres chanteront», Trio Fibonacci

Serge Provost, La pietra che canta

Wolfgang Rihm, Fremde Szenen

"Amours et rituels», Quatuor Bozzini

Jean Lesage, Quatuor $n^{\circ} 2$

Luigi Nono, Fragmente - Stille, An Diotima

\section{Mardi 11 mars 2003}

"Corps et Âmes», Orchestre symphonique de Montréal, Pierre-André Valade, dir.

Salle Pollack

Sean Ferguson, In the Flesh

Jean-Philippe Bec, Saturne

Michel Longtin, Quaternions*

* Création 

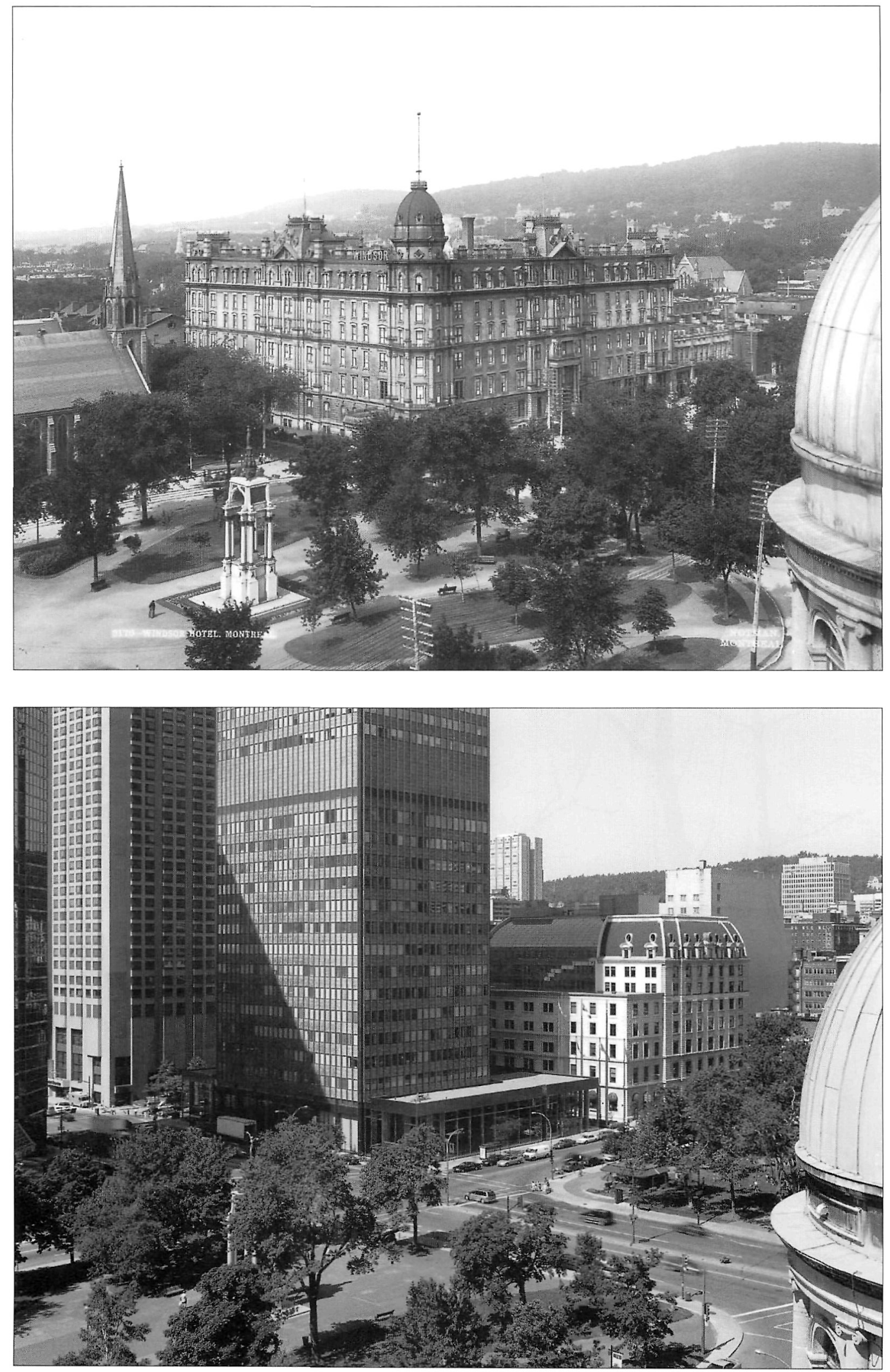

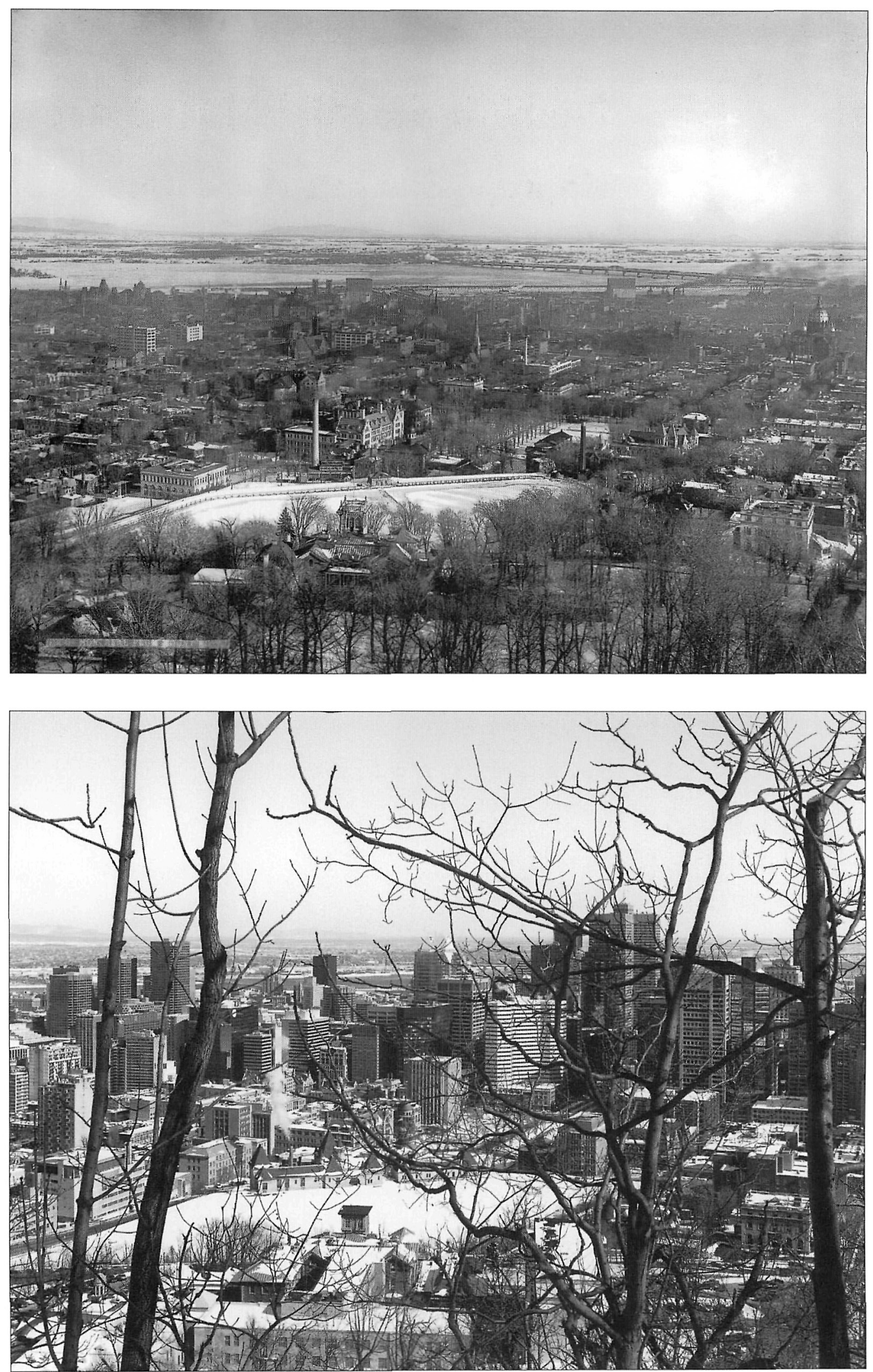